\title{
CEPAS NATIVAS DE Bacillus thuringiensis CONTRA Spodoptera frugiperda y Alabama argillacea EN EL CULTIVO DE ALGODÓN (Gossypium barbadens) EN PIURA, PERÚ
}

\author{
NATIVE STRAINS OF Bacillus thuringiensis AGAINST Spodoptera frugiperda \\ and Alabama argillacea IN COTTON CROP (Gossypium barbadens) IN PIURA, \\ PERU
}

\author{
Robert Rafael-Rutte ${ }^{1 *}$, René Aguilar², Edgar A. Maldonado², Marianella Ruiz² \\ ${ }^{1}$ Facultad de Ingeniería y Gestión, Universidad Nacional Tecnológica de Lima Sur, Sector 3 Grupo 1A 03 (Av. \\ Central y Av. Bolivar) Villa El Salvador C. P. 15834, Lima, Perú. \\ 2 Facultad de Agronomía, Departamento Académico de Sanidad Vegetal, Universidad Nacional de Piura, Urb. \\ Miraflores s/n, Castilla, C.P. 295, Piura, Perú. \\ * Autor para correspondencia E-mail: rrafael@untels.edu.pe
}

\section{RESUMEN}

Los lepidópteros son una de las plagas más importantes del algodón (Gossypium barbadens). Su control se realiza con insecticidas sintéticos, que contaminan el medio ambiente, por lo que el control biológico constituye una alternativa ecológica al uso de tales productos químicos. El objetivo de esta investigación fue aislar cepas nativas de Bacillus thuringiensis $(\mathrm{Bt}) \mathrm{y}$ evaluar su toxicidad contra Spodoptera frugiperda y Alabama argillacea, insectos plaga del cultivo de algodón. Las cepas nativas de Bt fueron aisladas de muestras de suelo de diferentes zonas algodoneras de Piura, Perú. Se obtuvieron 600 colonias del género Bacillus, de los cuales 37 cepas nativas presentaron características morfológicas similares a las cepas estándares de Bt (HD1 y NA118). La caracterización morfológica y bioquímica de las 37 cepas nativas mostró que 15 cepas presentaron cristal paraesporal de forma bipiramidal de acción tóxica contra lepidópteros, y características bioquímicas similares a las cepas estándares de Bt. El efecto tóxico de las 15 cepas nativas se evaluó con la prueba del alimento contaminado con discos de hojas de lechuga y algodón. Los resultados mostraron que las cepas IN24, IN-30 e IN-34 ocasionaron 100\% de mortalidad en $S$. frugiperda y las cepas IN-19, IN-24 e IN25 ocasionaron $100 \%$ de mortalidad en $A$. argillacea, mientras que la cepa IN-24 ocasionó $100 \%$ de mortalidad en ambas especies. Por otra parte, la prueba de la dieta artificial reveló que las cepas IN34, IN-01 e IN-31 ocasionaron los mayores porcentajes de mortalidad en S. frugiperda y A. argillacea, respectivamente. Se concluyó que existe varias cepas nativas de $B$. thuringiensis que pueden controlar S. frugiperda y A. argillacea.

Palabras claves: toxina, bacterias, bioinsecticida, lepidópteros, algodón

\section{ABSTRACT}

The order Lepidoptera is one of the most important pests of cotton (Gossypium barbadens). These insects are controlled with synthetic insecticides, which contaminate the environment. Therefore, biological control is an environmentally friendly alternative to the use of such chemicals. The objective of this research was to isolate native strains of Bacillus thuringiensis $(\mathrm{Bt})$ and evaluate their toxicity against Spodoptera frugiperda and Alabama argillacea, which are insect pests of the

Recibido: 27 septiembre 2019. Aceptado: 04 diciembre 2019. 
cotton crop. The native strains of Bt were isolated from soil samples from different cotton areas of the Piura, Perú. 600 colonies of the genus Bacillus were obtained, of which 37 native strains presented morphological characteristics similar to the standard strains of $B t$ (HD1 and NA118). The morphological and biochemical characterization showed that 15 strains showed parasporal crystal of bipyramidal form of toxic action against Lepidoptera, exhibiting biochemical characteristics equal to the standard strains of $B t$. The toxic effect of these 15 native strains was evaluated through a food poisoned test using lettuce and cotton leaf discs. The results showed that strains IN-24, IN-30 and IN-34 caused $100 \%$ mortality in S. frugiperda; strains IN-19, IN-24 and IN-25 caused $100 \%$ mortality in A. argillacea; while strain IN-24 caused $100 \%$ mortality in both species. The artificial diet test showed that strains IN-34, IN-01 and IN-31 caused the highest mortality rates in S. frugiperda and A. argillacea, respectively, when compared with the standard strains HD1 and NA118. It is concluded that there are several native strains of $B$. thuringiensis that can control S. frugiperda and A. argillacea.

Key words: toxin, bacteria, bioinsecticide, lepidoptera, cotton.

\section{INTRODUCCIÓN}

El algodón Gossypium barbadens, es el cultivo no alimenticio más sembrado a nivel mundial. En el Perú, es un insumo productivo para el sector textil y ha tenido siempre una gran relevancia social y económica. El algodón peruano es reconocido mundialmente por su calidad, ya que Perú es un centro de origen y domesticación de este cultivo, siendo Piura uno de sus departamentos productores, debido a que tiene condiciones agroecológicas adecuadas para el cultivo del algodón (Reyes, 2014; MINAGRI, 2019). Sin embargo, su producción $\mathrm{y}$ productividad ha presentado una tendencia fluctuante, debido al deterioro de la economía de los pequeños productores, a la falta de tecnología y capacitación, e inadecuado manejo agronómico (MINAGRI, 2019). Dentro de ello, uno de los factores limitantes en la producción son los insectos plaga, que limitan el incremento de la productividad en la mayoría de los países productores de algodón. En el caso de Piura, así como en otros países, los insectos plaga más importantes son los lepidópteros como el gusano cortador de plántulas Spodoptera frugiperda Smith (Noctuidae) (Casmuz et al., 2010), el gusano mayor de la hoja Alabama argillacea Hübner (Noctuidae) (Helman et al., 2011), el gusano menor de la hoja Anomys texana Riley (Noctuidae) y el gusano rosado de la India Pectinophora gossypiella Saunders (Gelechiidae), entre otras (Reyes, 2014).

Estos insectos ocasionan grandes pérdidas económicas a los productores algodoneros y como muchas otras plagas, el control principal se realiza mediante el uso de insecticidas químicos, los cuales contaminan el ambiente y ejercen una alta presión de selección sobre los insectos plaga que ocasionan una disminución en la eficiencia de las moléculas (Campos et al., 2016). Esto planteó la necesidad de desarrollar otras medidas de control basadas en otros compuestos o microorganismos, que controlen de forma eficaz las plagas presentes en los cultivos, que no dejen residuos en el campo, que no sean perjudiciales para el medio ambiente o salud humana, permitiendo al mismo tiempo el desarrollo de una agricultura sostenible (Souza et al., 2008).

En este sentido se han desarrollado insecticidas microbiológicos en base a la bacteria Bacillus thuringiensis (Bt) que es altamente comercializada a nivel mundial, por su gran toxicidad, alta especificidad, inocuidad para el medio ambiente y el humano, constituyéndose en una buena alternativa frente al uso indiscriminado de insecticidas químicos para el control de los insectos plaga (Vilas-Bôas et al., 2007; Bravo et al., 2010; Bravo et al., 2013; Adang et al., 2014). Bt puede ser aislada de diferentes hábitats, siendo el suelo el más común, la principal fuente natural de esporas y el sustrato preferido para el aislamiento de especies de Bacillus (Abdullah et al., 2014). Sin embargo, también se reportan aislamientos de ambientes acuáticos, insectos muertos, muestras de plantas y de silos (Iriarte et al., 2000; Alper et al., 2014; Alquisira et al., 2014).

El objetivo de este estudio fue aislar cepas nativas de Bacillus thuringiensis (Berliner) y evaluar su efecto toxico contra Spodoptera frugiperda y Alabama argillacea, insectos plaga del cultivo de algodón.

\section{MATERIALES Y MÉTODOS}

\section{Aislamiento e identificación de cepas nativas de Bacillus thuringiensis}

Se tomaron al azar 24 muestras de suelo agrícola de los Valles del Bajo $\left(5^{\circ} 16^{\prime} 56.14^{\prime \prime}\right.$; $\left.80^{\circ} 40^{\prime} 57.17^{\prime \prime} \mathrm{O}\right)$ y Medio ( $5^{\circ} 10^{\prime} 37.62^{\prime \prime} \mathrm{S} ; 80^{\circ} 0^{\prime} 1.72^{\prime \prime}$ O) Piura, zonas algodoneras localizadas en el departamento de Piura, Perú, a una profundidad de 15 a $20 \mathrm{~cm}$. Las muestras de suelo provenían de campos en los que no se habían realizado aplicaciones previas de insecticidas a base de B. thuringiensis. Las muestras se separaron, 
tamizaron y codificaron para ser procesados y analizadas de forma independiente. De cada una de las muestras procesadas se tomó $1 \mathrm{~g}$ que se mezcló con $10 \mathrm{~mL}$ de agua destilada estéril contenidas en tubos de ensayo, y se sometieron a una agitación rápida por $5 \mathrm{~min}$. Los tubos se incubaron en baño maría a $70^{\circ} \mathrm{C}$ durante 30 min con el objetivo de seleccionar las esporas presentes en la mezcla y tras un breve reposo, con una pipeta Eppendorff se tomaron $10 \mu \mathrm{L}$ de cada tubo para ser sembradas en placas de Petri conteniendo el medio esporulante CCY (Stewart et al., 1981), las cuales se incubaron a oscuridad (Isotemp ${ }^{\circledR}$ Incubator Fisher Scientific model 655D, Pittsburgh, Pensilvania, USA) por 72 horas a $28^{\circ} \mathrm{C}$ para lograr el crecimiento de las colonias de $B$. thuringiensis y seleccionarlas a simple vista por su aspecto y morfología. Ellas se distinguieron por ser de color blanco opaco con ciertas tonalidades grisáceas, de contornos irregulares y textura lisa y cerosa en el centro.

Las colonias bacterianas seleccionadas de $\mathrm{Bt}$, fueron sembradas en placas de Petri conteniendo medio de cutivo LB (Luria- Bertani) (Sambrook and Russell, 2001). Esta identificación y selección preliminar de las colonias se realizaron con microscopio óptico (Carl Zeiss Mod. KF 2, Oberkochen, Germany) y por la presencia de un cuerpo paraesporal. Las cepas seleccionadas anteriormente se sembraron nuevamente en placas de Petri que contenían medio CCY, incubadas por 24 horas y conservadas en glicerina al $5 \%$ y refrigeradas a $4{ }^{\circ} \mathrm{C}$ (Ruiz de Escudero, 2006). El aislamiento de las cepas nativas de $\mathrm{Bt}$ y los bioensayos se realizaron en el Laboratorio de Fitopatología de la Facultad de Agronomía de la Universidad Nacional de Piura.

\section{Producción y purificación de cristales de Bacillus thuringiensis}

Para la obtención del complejo espora-cristal, se realizó un preparado de $500 \mathrm{~mL}$ de medio CCY, a los que se les inoculó $1 \mathrm{~mL}$ de la cepa de $B$. thuringiensis aislada previamente. Los matraces Erlenmeyer se sometieron a una agitación constante por 72 horas en agitador horizontal. A los cultivos desarrollados se le añadieron 100 $\mathrm{mL}$ de $\mathrm{NaCl}(5 \mathrm{M})$ y se centrifugó 10 min a 10000 rpm y $4^{\circ} \mathrm{C}$. Esta mezcla de esporas y cristales se lavaron con agua destilada estéril, repitiéndose el lavado y centrifugado dos veces más. Los cristales y las esporas obtenidas como resultado de la centrifugación se recuperaron mediante una pipeta Pasteur, luego se almacenaron en tubos de $2 \mathrm{~mL}$ a $4^{\circ} \mathrm{C}$ para su posterior utilización (Ruiz de Escudero, 2006; Ruiz De Escudero et al., 2006).
Codificación y caracterización bioquímica de cepas nativas de Bacillus thuringiensis

La codificación de Bt se realizó basándose en la existencia y la morfología de la inclusión cristalina al lado de las esporas de cada una de las cepas aisladas, por ser uno de los criterios más utilizados en la caracterización de cepas de $B$. thuringiensis (Carreras, 2008).

La caracterización bioquímica de las 37 cepas nativas seleccionadas se hizo considerando las características bioquímicas comunes de B. thuringiensis. Se realizaron las pruebas bioquímicas de agar almidón, caldo nitrato, citrato simons, caldo ureasa, hidrólisis de gelatina, reducción indol, manitol, fermentación fructuosa, fermentación manosa, fermentación maltosa, catalasa y esculina; teniendo como patrón referente a los testigos positivos HD1 y NA118 (Ruiz De Escudero et al., 2004; 2006).

\section{Colección de insectos}

Los huevos, larvas y pupas de los insectos se recolectaron de plantas del cultivo de algodón $\mathrm{y}$ pimiento (Capsicum annuиm), no tratadas previamente con insecticidas sintéticos $\mathrm{o}$ biológicos, de los valles del Bajo y Medio Piura. Para S. frugiperda, se recolectaron masas de huevos y se colocaron en recipientes plásticos acondicionados para su eclosión. Para $A$. argillacea se recolectaron posturas y larvas, y se colocaron en cajas de plástico acondicionadas para su desarrollo, alimentándolas con brotes tiernos y hojas de algodón; las pupas se colocaron en una caja entomológica de $40 \mathrm{~cm}$ de largo por $30 \mathrm{~cm}$ de ancho y $33 \mathrm{~cm}$ de alto acondicionada en su interior con una botella pequeña conteniendo agua y brotes de algodón, para que las polillas recién emergidas coloquen sus huevos en los brotes para obtener nuevas generaciones y mayor cantidad de larvas juveniles.

\section{Bioensayos}

El efecto tóxico de las cepas nativas de $B$. thuringiensis se evaluó utilizando larvas de primer instar de $S$. frugiperda y $A$. argillacea de tamaño uniforme, mediante la dieta natural y artificial del alimento contaminado con las esporas y cristales de las cepas nativas, según la metodología descrita por Iriarte et al. (1998) y Sauka and Benintende (2017).

La alimentación de las larvas se realizó en placas de plástico de $13 \mathrm{~cm}$ de largo por $8,6 \mathrm{~cm}$ de ancho y $2,3 \mathrm{~cm}$ de altura divididos en 24 pocillos. En cada pocillo se introdujeron discos de hoja fresca de lechuga que fueron sembradas en el invernadero y algodón de $7 \mathrm{~mm}$ de diámetro previamente impregnados con esporas y cristales de las cepas de Bt y sobre estos se colocó una 
larva neonata, luego se cubrieron con doble papel absorbente y se hicieron 24 repeticiones por cepa. Las placas fueron tapadas, selladas con cintas adhesivas y colocadas en una caja de poliestireno a $25^{\circ} \mathrm{C}$. Las lecturas de mortalidad por cada tratamiento fueron a los cinco días (Iriarte et al., 1998; Sauka and Benintende 2017). Para obtener el porcentaje de mortalidad, se realizó el ajuste a los datos obtenidos mediante el Análisis Probit (Ibarra y Del Rincón, 2001).

La dieta artificial estuvo compuesta por germen de trigo, levadura de cerveza, caseína, azúcar, ácido sórbico, colesterol, aceite de lino, mezcla de sales y agar más esporas y cristales de cepas nativas de Bt en una proporción de 1:4 (esporas y cristales: dieta). Se utilizaron cajas cuadradas de plástico de $10 \mathrm{~cm}$ de largo por $10 \mathrm{~cm}$ de ancho y $2 \mathrm{~cm}$ de altura, dividida en 25 pocillos. En cada pocillo se colocaron porciones gelificadas de la dieta y sobre estos se colocó una larva neonata, y luego se cubrieron con doble papel absorbente. Las cajas se taparon, selladas con cinta adhesiva y mantenidas a $25^{\circ} \mathrm{C}$. Las lecturas de mortalidad por cada tratamiento fueron a los cinco días (Iriarte et al., 1998; Sauka and Benintende, 2017). Para obtener el porcentaje de mortalidad, se realizó el ajuste a los datos obtenidos mediante el Análisis Probit (Ibarra y Del Rincón, 2001).

Como controles positivos para las pruebas se utilizaron cepas estándares comerciales de $B$. thuringiensis de alta toxicidad, procedentes de España (HD1 y NA118). El testigo negativo fue agua destilada.

\section{RESULTADOS Y DISCUSION}

\section{Aislamiento de cepas nativas de Bacillus thuringiensis}

Se obtuvieron 600 colonias del género Bacillus, de los cuales 264 colonias (44\%) presentaron y desarrollaron características morfológicas similares a las observadas en las cepas estándares comerciales HD1 y NA118 de B. thuringiensis procedentes de España. Es decir, mostraron colonias circulares de color blanco, cremosas, con bordes irregulares y textura mucosa (Tabla 1).

Estos resultados concuerdan con las observaciones de Theunis et al. (1998), Iriarte et al. (1998), Schnepf et al. (1998), Salehi et al. (2008), Muniady et al. (2011), y Galvis y Moreno (2018), quienes en los diferentes trabajos realizados aislaron colonias con características morfológicas pertenecientes a $B$. thuringiensis.

\section{Codificación de cepas nativas de Bacillus thuringiensis}

La estructura paraesporal cristalina se observó en 37 aislados de los 600, que indican la presencia de $\mathrm{Bt}$, y la forma bipiramidal de esta inclusión paraesporal (Gitahy et al., 2007; Portela-Dussán et al., 2013; Salama et al., 2015) sólo se observó en 15 de los 37 aislados (Tabla 2), lo cual indicó que los aislados evaluados pertenecen al patotipo I de $B$. thuringiensis que son tóxicos para invertebrados, especialmente para larvas de los insectos de la orden Lepidoptera (Schnepf et al., 1998; Carreras, 2008; Sauka et al., 2010), Coleoptera y Diptera, entre otros (Portela-Dussán et al., 2013; Adang et al., 2014; Palma et al., 2014; Reyaz et al., 2017), razón por el cual se evaluó en larvas del primer instar de S. frugiperda y A. argillacea.

Estos resultados coincidieron con Ruiz De Escudero et al. (2004) y Rosas-Garcia et al. (2008), que observaron mayor cantidad (89\%) de cristales de forma bipiramidal en comparación que cualquier otra morfología. También concuerdan con otros autores (Höfte y Whitley, 1989; Baró et al., 2009; Ramírez et al., 2010; Azizoglu et al., 2011; Vázquez-Ramírez et al., 2015), quienes al analizar cepas nativas observaron una morfología de cristales bipiramidales típicos de la mayoría de las cepas pertenecientes a $B$. thuringiensis (Rabinovich et al. 2017).

La codificación se realizó de acuerdo a como se iban encontrando las colonias de $B$. thuringiensis con distintas formas del cristal paraesporal en los aislados observados. Sin embargo, estos cristales pueden presentar distintas morfologías y ser clasificados en bipiramidales, cúbicos, cuadrados, aplanados, esféricos y otras formas

Tabla 1. Aislamiento de cepas nativas de B. thuringiensis de diferentes zonas algodoneras de Piura, Perú. 2018.

Table 1. Isolation of native strains of B. thuringiensis from different cotton areas de Piura, Perú. 2018.

\begin{tabular}{lcccc}
\hline Zonas de muestreo & $\begin{array}{c}\text { Muestras } \\
\text { analizadas }\end{array}$ & $\begin{array}{c}\text { Colonias } \\
\text { aisladas }\end{array}$ & $\begin{array}{c}\text { Coloración } \\
\text { Gram } \\
\text { positivo }\end{array}$ & $\begin{array}{c}\text { Colonias con } \\
\text { características } \\
\text { de Bt }\end{array}$ \\
\hline Bajo Piura & 12 & 300 & 132 & 2 \\
Medio Piura & 12 & 300 & 132 & 13 \\
TOTAL & 24 & 600 & 264 & 15 \\
\hline
\end{tabular}


Tabla 2. Codificación de cepas nativas de Bacillus thuringiensis aisladas de las zonas algodoneras de Piura, Perú.

Table 2. Coding of native strains of Bacillus thuringiensis isolated from the cotton areas of Piura, Perú.

\begin{tabular}{|c|c|c|c|c|}
\hline $\begin{array}{c}\mathrm{N}^{\circ} \text { de } \\
\text { Aislamientos }\end{array}$ & Código & Tinción Gram & $\begin{array}{c}\text { Presencia de } \\
\text { posibles cristales }\end{array}$ & $\begin{array}{c}\text { Cristales de } \\
\text { forma piramida }\end{array}$ \\
\hline 1 & IN-01 & + & + & si \\
\hline 2 & IN-02 & + & + & si \\
\hline 3 & IN-03 & + & + & no \\
\hline 4 & IN-04 & + & + & no \\
\hline 5 & IN-05 & + & + & no \\
\hline 6 & IN-06 & + & + & no \\
\hline 7 & IN-07 & + & + & no \\
\hline 8 & IN-08 & + & + & no \\
\hline 9 & IN-09 & + & + & no \\
\hline 10 & IN-10 & + & + & no \\
\hline 11 & IN-11 & + & + & no \\
\hline 12 & IN-12 & + & + & no \\
\hline 13 & IN-13 & + & + & no \\
\hline 14 & IN-14 & + & + & no \\
\hline 15 & IN-15 & + & + & no \\
\hline 16 & IN-16 & + & + & no \\
\hline 17 & IN-17 & + & + & no \\
\hline 18 & IN-18 & + & + & no \\
\hline 19 & IN-19 & + & + & si \\
\hline 20 & IN-20 & + & + & no \\
\hline 21 & IN-21 & + & + & si \\
\hline 22 & IN-22 & + & + & si \\
\hline 23 & IN-23 & + & + & si \\
\hline 24 & IN-24 & + & + & si \\
\hline 25 & IN-25 & + & + & si \\
\hline 26 & IN-26 & + & + & si \\
\hline 27 & IN-27 & + & + & si \\
\hline 28 & IN-28 & + & + & no \\
\hline 29 & IN-29 & + & + & no \\
\hline 30 & IN-30 & + & + & si \\
\hline 31 & IN-31 & + & + & si \\
\hline 32 & IN-32 & + & + & si \\
\hline 33 & IN-33 & + & + & no \\
\hline 34 & IN-34 & + & + & si \\
\hline 35 & IN-35 & + & + & no \\
\hline 36 & IN-36 & + & + & no \\
\hline 37 & IN-37 & + & + & si \\
\hline
\end{tabular}

atípicas menos frecuentes (Schnepf et al., 1998; Monnerat et al., 2007; Sauka y Benintende, 2008; Santos et al., 2009; Ramírez et al., 2010; Ricieto et al., 2013), encontrándose en el presente trabajo células vegetativas, endosporas y cristales de forma piramidal.

\section{Caracterización bioquímica}

Las 15 cepas nativas seleccionadas con presencia del cristal bipiramidal presentaron en general las mismas características bioquímicas de los testigos positivos HD1 y NA118 (Tabla 3), es decir presentaban la capacidad de fermentar fructosa, manosa y maltosa, y de hidrolizar gelatina, almidón y esculina (Ruiz De Escudero, 2006).

Los resultados de estas pruebas concuerdan con lo señalado por Lecadet et al. (1999), 
Tabla 3. Caracterización bioquímica de cepas nativas de Bacillus thuringiensis aisladas de las zonas algodoneras de Piura, Perú.

Table 3. Biochemical characterization of native strains of Bacillus thuringiensis isolated from the cotton areas of Piura, Perú.

\begin{tabular}{lllllllllllllll}
\hline CEPAS & TG & C & AA & CN & CS & CU & HG & RI & M & FF & FM & FM & E \\
\hline HD1 & + & + & + & + & - & - & + & - & - & + & + & + & + \\
NA118 & + & + & + & + & - & - & + & - & - & + & + & + & + \\
IN-01 & + & + & + & + & - & - & + & - & - & + & + & + & + \\
IN-02 & + & + & + & + & - & - & + & - & - & + & + & + & + \\
IN-03 & + & + & - & + & + & - & + & - & + & + & + & + & + \\
IN-04 & + & + & + & - & + & - & + & - & + & + & - & + & + \\
IN-05 & + & + & + & - & + & - & + & - & + & + & - & + & + \\
IN-06 & + & + & + & - & - & - & + & - & + & + & + & + & + \\
IN-12 & + & + & + & + & + & - & + & - & + & + & + & + & + \\
IN-15 & + & + & + & - & + & - & + & - & + & + & - & + & + \\
IN-16 & + & + & + & - & + & - & + & - & + & + & + & + & + \\
IN-17 & + & + & + & - & + & - & + & - & + & + & - & + & + \\
IN-18 & + & + & + & - & + & - & + & - & + & + & - & + & + \\
IN-19 & + & + & + & + & - & - & + & - & - & + & + & + & + \\
IN-20 & + & + & + & + & - & - & + & - & - & + & + & + & + \\
IN-21 & + & + & + & + & + & - & + & - & + & + & + & + & + \\
IN-22 & + & + & + & + & - & - & + & - & - & + & + & + & + \\
IN-23 & + & + & + & + & - & - & + & - & - & + & + & + & + \\
IN-24 & + & + & + & + & - & - & + & - & - & + & + & + & + \\
IN-25 & + & + & + & + & - & - & + & - & - & + & + & + & + \\
IN-26 & + & + & + & + & - & - & + & - & - & + & + & + & + \\
IN-27 & + & + & + & + & - & - & + & - & - & + & + & + & + \\
IN-28 & + & + & + & + & - & + & + & - & - & + & + & + & + \\
IN-29 & + & + & + & + & - & + & + & - & + & + & + & + & + \\
IN-30 & + & + & + & + & - & - & + & - & - & + & + & + & + \\
IN-31 & + & + & + & + & - & - & + & - & - & + & + & + & + \\
IN-32 & + & + & + & + & - & - & + & - & - & + & + & + & + \\
IN-33 & + & + & + & + & - & + & + & - & - & + & + & + & + \\
IN-34 & + & + & + & + & - & - & + & - & - & + & + & + & + \\
IN-35 & + & + & + & + & - & + & + & - & + & + & + & + & + \\
IN-36 & + & + & + & + & + & - & + & - & - & + & + & + & + \\
IN-37 & + & + & + & + & - & - & + & - & - & + & + & + & + \\
\hline Z & + & + & +
\end{tabular}

Zona coloreada $=$ Cepas bioquímicamente idénticas con los testigos positivos HD1 y NA 118); TG = Tinción Gram, $\mathrm{C}=$ Catalasa, $\mathrm{AA}=$ Agar Almidón, $\mathrm{CN}=$ Caldo Nitrato, $\mathrm{CS}=$ Citrato Simons, $\mathrm{CU}=$ Caldo Ureasa, $\mathrm{HG}=$ Hidrolisis de gelatina, $\mathrm{RI}=$ Reducción Indol, $\mathrm{M}=$ Manitol, $\mathrm{FF}=$ Fermentación Fructuosa, FM = Fermentación Manosa, Fm = Fermentación maltosa, E = Esculina.

Martin et al. (2010) y Blackburn et al. (2013) quienes analizaron y evaluaron una serie de características bioquímicas $(\mathrm{ADH}$, enzimas para reducir nitratos, ureasa, habilidad para fermentar sucrosa, manosa, celobiosa, esculina y producir AMC) para diferenciar e identificar grupos de serovars y cepas bacterianas de B. thuringiensis, y también con Ruiz De Escudero (2006), quien caracterizó bioquímicamente la cepa Bt NA118 y demostró que es capaz de fermentar D-ribosa,
D-glucosa, D-fructosa, N-acetilglucosamina, arbutina, esculina/citrato férrico, salicina, D-celobiosa, D-maltosa, D-trehalosa, almidón y glucógeno y que tenía capacidad para utilizar 49 azúcares y fermentarlos de acuerdo al test API 50 CH (API System, BioMérieux S.A., Marcy I'Etoile, FR) (Fig. 1).

Estos resultados de las pruebas bioquímicas también se asemejan a los datos reportados por otros autores para B. thuringiensis, en la 

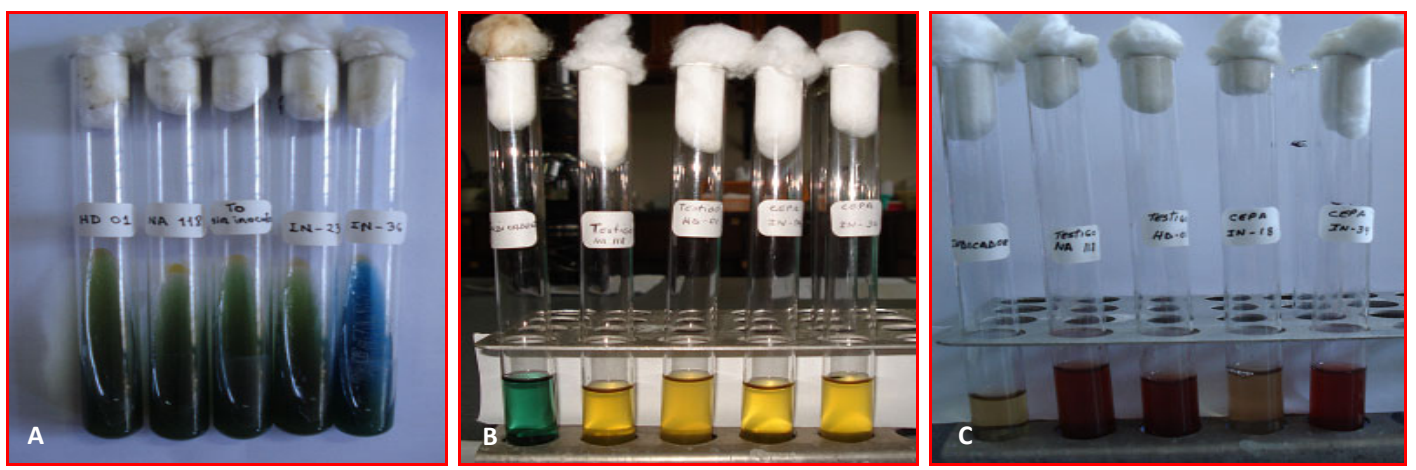

Fig. 1. Pruebas bioquímicas. A) Citrato Simons, negativo para las cepas nativas de Bt; B) Fermentación de fructuosa, positivo para las cepas nativas de Bt; y C) Prueba de reducción de nitratos positivas para las cepas nativas de Bt.

Fig. 1. Biochemical tests. A) Citrato simons, negative for native strains of Bt; B) Fructose fermentation, positive for native strains of $\mathrm{Bt}$; and $\mathrm{C}$ ) Positive nitrate reduction test for native strains of $\mathrm{Bt}$.

que demuestran que Bt es un microorganismo con actividad de catalasa (Schnepf et al., 1998; Alvarez et al., 2011) y que las distintas cepas de B. thuringiensis poseen la capacidad de fermentar glucosa, fructosa, trehalosa, maltosa y ribosa, y de hidrolizar gelatina, almidón, glucógeno, esculina y Nacetil-glucosamina (Sauka y Benintende, 2008; Blackburn et al., 2013).

\section{Bioensayos}

Los tipos de bioensayo utilizados permitieron determinar cuantitativamente la toxicidad de las cepas aisladas contra los insectos de la orden Lepidóptero $S$. frugiperda y $A$. argillacea. Los ensayos se realizaron a partir de concentraciones del complejo espora más cristales.

\section{Toxicidad con discos de hojas de lechuga y algodón}

En la prueba del alimento contaminado de discos de hojas de lechuga y algodón, se observó que las 15 cepas nativas (con presencia del cristal bipiramidal) presentaron efectos tóxicos $\geq$ al $70 \%$ contra $S$. frugiperda $\mathrm{y} \geq 5 \%$ contra $A$. argillacea (Tabla 4). Las cepas IN-24, IN-30 e IN-34 ocasionaron $100 \%$ de mortalidad en S. frugiperda, seguidos por las cepas IN-19, IN-23 e IN-37 que ocasionaron $91,67 \%$ de mortalidad. Mientras que las cepas IN-19, IN-24 e IN-25 ocasionaron 100\% de mortalidad de $A$. argillacea, seguidos por las cepas IN-31 e IN-34 que presentaron un 95,83\% de mortalidad (Tabla 4). Es importante señalar que la cepa IN-24 causó $100 \%$ de mortalidad en ambas especies evaluadas, seguido de las cepas IN-19 e IN-34 que ocasionaron más del 90\% de mortalidad (Tabla 4).

El alto efecto tóxico de las cepas nativas aisladas contra los lepidópteros evaluados, probablemente sea porque las larvas neonatas se alimentaron con su propio hospedante, en este caso lechuga y algodón. Para S. frugiperda los resultados obtenidos pueden deberse a su tipo de alimentación, ya que al ser un insecto voraz con comportamiento fágico caníbal, las larvas neonatas consumieron más área foliar impregnada con toxinas; a diferencia de las larvas neonatas de $A$. argillacea, que consumen poco alimento en sus primeros estadíos (Johnson, 1984).

\section{Toxicidad con la dieta artificial}

En la prueba de la dieta artificial contaminada, se observó que las 15 cepas nativas presentaron baja actividad tóxica contra S. frugiperda y $A$. argillacea (Tabla 5). Las cepas que ocasionaron los mayores porcentajes de mortalidad en $S$. frugiperda fueron IN-34 (79,17\%), IN-01 e IN-31 ambos con $62,50 \%$ de mortalidad, seguidos de las cepas IN-37, IN-02 e IN-19 con más del 45\% de mortalidad (Tabla 5). Mientras que las cepas que ocasionaron los mayores porcentajes de mortalidad en $A$. argillacea fueron IN-01 (66,67\%), IN-34 (45,12\%) e IN-31 (40,50\%), seguidos de las cepas IN-02 (37,50\%), IN-23 (33.33\%) e IN-37 $(32,65 \%)$ (Tabla 5).

El efecto tóxico relativamente bajo de las cepas nativas aisladas puede deberse al tipo de la dieta artificial que no fue agradable para estos insectos o también, es posible que los insectos no fueron susceptibles (Martín y Travers, 1989; Iriarte et al., 1998).

Los resultados del presente trabajo difieren con los obtenidos por Ruiz De Escudero (2006), quien reporta al testigo HD1 con una baja acción tóxica contra S. frugiperda, siendo lo contrario en nuestros bioensayos, donde la misma cepa 
Tabla 4. Toxicidad contra Spodoptera frugiperda y Alabama argillace obtenida mediante la prueba del alimento contaminado con discos de hojas de lechuga y algodón.

Table 4. Toxicity against Spodoptera frugiperda and Alabama argillace obtained by the food poisoning test using lettuce and cotton leaf discs.

\begin{tabular}{lcccc}
\hline \multirow{2}{*}{ CEPAS } & \multicolumn{2}{c}{ Spodoptera frugiperda } & \multicolumn{2}{c}{ Alabama argillacea } \\
\cline { 2 - 5 } & $\begin{array}{c}\text { Larvas vivas } \\
\mathbf{( \% )}\end{array}$ & $\begin{array}{c}\text { Larvas muertas } \\
\mathbf{( \% )}\end{array}$ & $\begin{array}{c}\text { Larvas vivas } \\
\mathbf{( \% )}\end{array}$ & $\begin{array}{c}\text { Larvas muertas } \\
\mathbf{( \% )}\end{array}$ \\
\hline Testigo (-) & 87,50 & 12,50 & 79,17 & 20,83 \\
HD1 & 29,17 & 70,83 & 41,67 & 58,33 \\
NA118 & 20,83 & 79,17 & 37,50 & 62,50 \\
IN-01 & 25,00 & 75,00 & 8,33 & 91,67 \\
IN-02 & 45,83 & 54,17 & 54,17 & 45,83 \\
IN-19 & 8,33 & 91,67 & 0,00 & 100,00 \\
IN-20 & 29,17 & 70,83 & 16,67 & 83,33 \\
IN-22 & 16,67 & 83,33 & 12,50 & 87,50 \\
IN-23 & 8,33 & 91,67 & 29,17 & 70,83 \\
IN-24 & 0,00 & 100,00 & 0,00 & 100,00 \\
IN-25 & 16,67 & 83,33 & 0,00 & 100,00 \\
IN-26 & 16,67 & 83,33 & 8,33 & 91,67 \\
IN-27 & 37,50 & 62,50 & 37,50 & 62,50 \\
IN-30 & 0,00 & 100,00 & 70,83 & 29,17 \\
IN-31 & 12,50 & 87,50 & 4,17 & 95,83 \\
IN-32 & 16,67 & 83,33 & 87,50 & 12,50 \\
IN-34 & 0,00 & 100,00 & 4,17 & 95,83 \\
IN-37 & 8,33 & 91,67 & 50,00 & 50,00 \\
\hline
\end{tabular}

Tabla 5. Toxicidad contra Spodoptera frugiperda y Alabama argillace obtenida mediante el método de la dieta artificial.

Table 5. Toxicity against Spodoptera frugiperda and Alabama argillace obtained by the artificial diet method.

\begin{tabular}{lcccc}
\hline \multirow{2}{*}{ CEPAS } & \multicolumn{2}{c}{ Spodoptera frugiperda } & \multicolumn{2}{c}{ Alabama argillacea } \\
\cline { 2 - 5 } & $\begin{array}{c}\text { Larvas vivas } \\
\mathbf{( \% )}\end{array}$ & $\begin{array}{c}\text { Larvas muertas } \\
\mathbf{( \% )}\end{array}$ & $\begin{array}{c}\text { Larvas vivas } \\
\mathbf{( \% )}\end{array}$ & $\begin{array}{c}\text { Larvas muertas } \\
\mathbf{( \% )}\end{array}$ \\
\hline Testigo (-) & 91,66 & 8,34 & 79,17 & 20,83 \\
HD1 & 70,33 & 29,87 & 66,67 & 33,33 \\
NA118 & 41,67 & 58,33 & 62,50 & 37,50 \\
IN-01 & 37,50 & 62,50 & 33,33 & 66,67 \\
IN-02 & 50,00 & 50,00 & 62,50 & 37,50 \\
IN-19 & 54,17 & 45,83 & 87,50 & 12,50 \\
IN-20 & 87,50 & 12,50 & 79,17 & 20,83 \\
IN-22 & 100,00 & No tóxica & 95,83 & 4,17 \\
IN-23 & 70,00 & 30,00 & 66,67 & 33,33 \\
IN-24 & 75,00 & 25,00 & 70,83 & 29,17 \\
IN-25 & 91,67 & 8,33 & 87,50 & 12,50 \\
IN-26 & 100,00 & No tóxica & 100,00 & No tóxica \\
IN-27 & 100,00 & No toxica & 86,13 & 13,87 \\
IN-30 & 87,50 & 12,50 & 91,67 & 8,33 \\
IN-31 & 37,50 & 62,50 & 59,50 & 40,50 \\
IN-32 & 83,33 & 16,67 & 92,50 & 7,50 \\
IN-34 & 20,83 & 79,17 & 54,88 & 45,12 \\
IN-37 & 41,67 & 58,33 & 67,35 & 32,65 \\
\hline
\end{tabular}


presentó una mayor toxicidad ocasionando un $70,83 \%$ de mortalidad de $S$. frugiperda. Los resultados de toxicidad de Bt sobre $A$. argillacea, no pueden ser contrastados con otros datos por no encontrarse publicaciones similares al trabajo realizado.

Los datos obtenidos con los protocolos empleados también permiten asumir que el tipo de alimento empleado juega un rol importante en los resultados de estas pruebas. El potencial insecticida encontrado en las cepas nativas aisladas de $B$. thuringiensis contra los lepidópteros evaluados son similares a las cepas obtenidas en los diferentes lugares del mundo, en la que también se encontró cepas con alta acción toxica que pueden ser usados como ingredientes activos para la elaboración de productos comerciales (Iriarte et al. 1998; Hernandez-Rodríguez y Ferré, 2009).

Los bioensayos cualitativos contra las larvas de primer instar de $S$. frugiperda y A. argillacea utilizando cepas nativas de $B$. thuringiensis, manifestaron niveles de toxicidad equivalentes a los de los controles positivos HD1 y NA118. Además, los resultados encontrados sugieren la existencia en Piura, Perú, de cepas nativas de Bt con alta toxicidad hacia $S$. frugiperda y $A$. argillacea.

\section{CONCLUSIONES}

Se concluyó que existe suficiente potencial de cepas nativas de $B$. thuringiensis con alta toxicidad para el control de $S$. frugiperda y A. argillacea, el cual le da a Piura una ventaja competitiva en el cultivo de algodón.

Las cepas nativas aisladas que ocasionaron mayor efecto tóxico contra $S$. frugiperda y $A$. argillacea fueron IN-24, IN-34, IN-19, IN-25, IN-30 e IN-31.

\section{LITERATURA CITADA}

Abdullah, R.R., N.A. Sukar, and N.M. Ghanim. 2014. Improving efficacy of Bacillus thuringiensis against insects of different feeding habits by plasmid transfer technique of chitinase. Egypt. J. Biol. Pest Control 24(1):37-44.

Adang, M.J., N. Crickmore, and J.L. Jurat-Fuentes. 2014. Diversity de Bacillus thuringiensis crystal toxins and mechanism of action. $p$. 39-87. In D. Tarlochan and G. Sarjeet (eds.) Advances in Insect Physiology 47. Academic Press, Oxford, USA.
Alper, M., H. Güneş, A. Tatlipinar, B. Çöl, H. Civelek, C. Özkan, and B. Poyraz. 2014. Distribution, occurrence of cry genes, and lepidopteran toxicity of native Bacillus thuringiensis isolated from fig tree environments in Aydin Province. Turk. J. Agric. For. 38(6):898-907. doi: 10.3906/tar1402-27.

Alquisira-Ramírez, E.V., J.R. Paredes-Gonzalez, V.M. Hernández-Velázquez, J.A. RamírezTrujillo, and G. Peña-Chora. 2014. In vitro susceptibility of Varroa destructor and Apis mellifera to native strains of Bacillus thuringiensis. Apidologie 45(6):707-718. doi: 10.1007/s13592-014-0288-z.

Alvarez, A., E. Virla, L. Pera, and M. Baigorí. 2011. Biological characterization of two Bacillus thuringiensis strains toxic against Spodoptera frugiperda. World Journal of Microbiology and Biotechnology 27:2343-2349. doi:10.1007/ s11274-011-0701-y.

Azizoglu, U., S. Yilmaz, A. Ayvaz, S. Karaborklu, and M. Akbulut. 2011. Characterization of local Bacillus thuringiensis isolates and their toxicity to Ephestia kuehniella (Zeller) and Plodia interpunctella (Hubner) larvae. Egyptian Journal of Biological Pest Control 21(2):143150.

Baró, Y., D. Fontana, y R.D. Santos. 2009. Caracterización de cepas de Bacillus thuringiensis Berliner y actividad biológica hacia Spodoptera frugiperda (J.E. Smith) (Lepidoptera: Noctuidae) y Anticarsia gemmatalis Hübner (Lepidoptera: Noctuidae). Fitosanidad 13(3):187-192.

Blackburn M.B., P.A. Martin, D. Kuhar, R.R. Jr. Farrar, and D.E. Gundersen-Rindal. 2013. Phylogenetic distribution of phenotypic traits in Bacillus thuringiensis determined by multilocus sequence analysis. PLoS One. 8(6):1-5. doi:10.1371/journal.pone.0066061.

Bravo, A., S. Gill, and M. Soberón. 2010. Bacillus thuringiensis: Mechanisms and use. p. 247277. In G. Lawrence and G. Sarjeet (eds.) Insect Control Biological and Synthetic Agents. Academic Press, London, USA.

Bravo, A., I. Gómez, H. Porta, B. García-Gómez, C. Rodriguez -Almanzan, L. Pardo, and M. Soberón. 2013. Evolution of Bacillus thuringiensis Cry toxins insecticidal activity. Microb. Biotechnol. 6(1):17-26. doi:10.1111/ j.1751-7915.2012.00342.x

Campos, E.V., J.L. de Oliveira, M. Pascoli, R. de Lima, and L.F. Fraceto. 2016. Neem oil and crop protection: from now to the future. Front. Plant Sci. 7:14-94. doi: 10.3389/ fpls.2016.01494. 
Carreras, B., S. Rodríguez, O. Ancheta, T. Valdés, y Y. Harvey. 2008. La morfología de los cuerpos paraesporales y su relación con la actividad biológica en cepas cubanas de Bacillus thuringiensis. Revista CENIC Ciencias Biológicas 39(2):105-108.

Casmuz, A., M.L. Juárez, M.G. Socias, M.G. Murúa, S. Prieto, S. Medina, E. Willink, y G. Gastaminza. 2010. Revisión de los hospederos del gusano cogollero del maíz Spodoptera frugiperda (Lepidoptera: Noctuidae). Rev. Soc. Entomol. Argent. 69(3-4):209-231.

Galvis, F., y L. Moreno. 2018. Identification of Cry1 genes in Bacillus thuringiensis isolates and their toxic effect against Milax gagates, a pest on lettuce (Lactuca sativa). Revista Chapingo Serie Horticultura 24(2):97-106. doi:10.5154/r. rchsh.2017.07.025.

Gitahy, P.D.M., M.T.D. Souza, R.G. Monnerat, E.D.B. Arrigoni, and J.I. Baldani. 2007. A Brazilian Bacillus thuringiensis strain highly active to sugarcane borer Diatraea saccharalis (Lepidoptera: Crambidae). Brazilian Journal Microbiology 38:531-537.

Helman, S.A., R.E. Beltran, F. Garay, y E. Raña. 2011. Efecto de las poblaciones de Alabama argillacea (Hübner) (Lepidoptera: Noctuidae) en cultivos de algodón a dos distanciamientos. Neotrop. Entomol. 40(2):251-257.

Hernández-Rodríguez, C.S., and J. Ferré. 2009. Ecological distribution and characterization of four collections of Bacillus thuringiensis strains. J. Basic Microbiol. 49(2):152-157. doi:10.1002/jobm.200800121.

Höfte, H., and H.R. Whiteley. 1989. Insecticidal crystal proteins of Bacillus thuringiensis. Microbiol. Rev. 53:242-255.

Ibarra, J., y M.C. Del Rincón. 2001. Cuantificación toxicológica de Bacillus thuringiensis. p. 133-152. En P. Caballero, J. Ferré (eds.) Bioinsecticidas: fundamentos y aplicaciones de Bacillus thuringiensis en el control integrado de plagas. Phytoma, Navarra, España.

Iriarte, J., Y. Bel, M.D. Ferrandis, R. Andrew, J. Murillo, J. Ferré, et al. 1998. Enviromental distribution and diversity of Bacillus thuringiensis in Spain. Syst. Appl. Microbiol. 21:97-106

Iriarte, J., M. Porcar, M.M. Lecadet, and P. Caballero. 2000. Isolation and characterization of Bacillus thuringiensis strains from aquatic environments in Spain. Current Microbiology 40: 402-408.

Johnson, S.J. 1984. Larval development, consumption and feeding behavior of the cotton leaf worm $A$. argillacea $\mathrm{H}$. The Southwestern Entomologist Louisiana. State University Agricultura Center 9(1):1-5.
Lecadet, M.M., E. Frachon, V.C. Dumanoir, H. Ripouteau, S. Hamon, P. Laurent, et al. 1999. Updating the $\mathrm{H}$-antigen classification of Bacillus thuringiensis. J. Appl. Microbiol. 86:660-672.

Martin, P.A., D.E. Gundersen, and M.B. Blackburn. 2010. Distribution of phenotypes among Bacillus thuringiensis strains. Systematic and Applied Microbiology 33:204-208. doi: 10.1016/j.syapm.2010.04.002.

Martin, P.A.W., and R.S. Travers. 1989. Worldwide abundance and distribution of Bacillus thuringiensis isolates. Appl. Environ. Microbiol. 55: 2437-2442.

Ministerio de Agricultura y Riego - MINAGRI. 2019. El Algodón. Dirección General de Políticas Agrarias, Lima, Perú.

Monnerat, R.G., A.C. Batista, P.T. Medeiros, E. Martins, V. Melatti, L. Praça, et al. 2007. Characterization of Brazilian Bacilus thuringiensis strains active against Spodoptera frugiperda, Plutella xylostella and Anticarsia gemmatalis. Biological Control 41:291-295.

Muniady, S., X. Rathinam, and S. Subramaniam. 2011. Quick isolation and characterization for the confirmation of a novel Bacillus thuringiensis strains from chicken manure samples. African Journal of Microbiology Research 5(20):3131-3137. doi:10.5897/ AJMR10.649

Palma, L., D. Muñoz, C. Berry, J. Murillo, and P. Caballero. 2014. Bacillus thuringiensis toxins: An overview of their biocidal activity. Toxins 6:3296-3325. doi:10.3390/toxins6123296.

Portela-Dussán, D.D., A. Chaparro-Giraldo, y S. López-Pazos. 2013. La biotecnología de Bacillus thuringiensis en la agricultura. Ciencias Biomédicas 11:87-96.

Rabinovitch, L., A. Vivoni, V. Machado, N. Knaak, D. Berlitz, R. Polanczyk, et al. 2017. Bacillus thuringiensis Characterization: Morphology, physiology, biochemistry, pathotype, cellular, and molecular aspects. p. 1-18. In L. Fiuza, R. Polanczyk, N. Crickmore (eds). Bacillus thuringiensis and Lysinibacillus sphaericus. Springer International Publishing AG, Cham, Switzerland. doi:https://doi. org/10.1007/978-3-319-56678-8

Ramírez, L., N. Ramírez, L.S. Fuentes, J. Jiménez, y J. Hernández. 2010. Aislamiento y caracterización molecular y biológica de cepas nativas de Bacillus thuringiensis para el control de Tuta absoluta (Meyrick: Lepidoptera: Gelechiidae), insecto plaga del tomate (Lycopersicon esculentum). Rev. Asoc. Col. Cienc. 22:73-96. 
Reyaz, A.L., L. Gunapriya, and P. IndraArulselvi. 2017. Molecular characterization of indigenous Bacillus thuringiensis strains isolated from Kashmir Valley. 3 Biotech 7:143 doi: 10.1007/s13205-017-0756-z.

Reyes, P. 2014. El algodón pima peruano: Cultivo y manejo agronómico. Universidad Nacional Piura, Chiclayo, Perú.

Ricieto, A.P., F.A. Fazion, C.D. Carvalho Filho, L.A. Vilas-Boas, and G.T. Vilas-Bôas. 2013. Effect of vegetation on the presence and genetic diversity of Bacillus thuringiensis in soil. Canadian Journal of Microbiology 59(1):28-33. doi: 0.1139/cjm-2012-0462.

Rosas-Garcia, N.M., M. Mireles-Martinez, J.L. Hernández-Mendoza, and J.E. Ibarra. 2008. Screening of cry gene contents of Bacillus thuringiensis strains isolated from avocado orchards in Mexico, and their insecticidal activity towards Argyrotaenia sp. (Lepidoptera: (Lepidoptera: Tortricidae) Larvae. Journal of Applied Microbiology. 104:224-230.

Ruiz De Escudero, I., M.A. Padilla, A.A. Ibáñez, y P. Caballero. 2004. Aislamiento y caracterización de nuevas cepas de Bacillus thuringiensis procedentes de muestras de tierra de Canarias. Bol. San. Veg. Plagas. España 30:703-712.

Ruiz De Escudero, I. 2006. Bacillus thuringiensis (Berliner) toxins for the control of lepidopteran pests. Memoria de Tesis para optar al grado de Doctor. Universidad Pública de Navarra, Navarra, España.

Ruiz De Escudero, I., A. Estela, M. Porcar, C. Martinez, J. A. Oguiza, B. Escriche, et al. 2006. Molecular and insecticidal characterization of a Cry1 I protein toxic to insects of the families Noctuidae, Tortricidae, Plutellidae, and Chrysomelidae. Appl. Environ. Microbiol. 72(7):4796-4804.

Salama, H.S., N.M. Abd El-Ghany, and M.M. Saker. 2015. Diversity of Bacillus thuringiensis isolates from Egyptian soils as shown by molecular characterization. Journal of Genetic Engineering and Biotechnology 13:101-109. doi:10.1016/j.jgeb.2015.10.001.

Salehi, G., A. Pourjan, A. Seifinejad, R. Marzban, K. Kariman, and B. Maleki. 2008. Distribution and diversity of dipteran specific cry and cyt genes in native Bacillus thuringiensis strains obtained from different ecosystems of Iran. J. Ind. Microbiol. Biotechnol. 35:83-94. doi:10.1007/s10295-007-0269-6.

Sambrook, J., and R.W. Russell. 2001. Molecular cloning: A laboratory manual. 3th ed. Cold Spring Harbor Laboratory Press, Cold Spring Harbor, New York, USA.
Santos, K.B.D., P. Neves, A.M. Meneguim, R.B.D. Santos, W.J.D. Santos, G. Villas-Boas, et al. 2009. Selection and characterization of the Bacillus thuringiensis strains toxic to Spodoptera eridania (Cramer), Spodoptera cosmioides (Walker) and Spodoptera frugiperda (Smith) (Lepidoptera: Noctuidae). Biological Control 50(2):157-163. doi:10.1016/j. biocontrol.2009.03.014.

Sauka, D.H., y G.B. Benintende. 2008. Bacillus thuringiensis: Generalidades. Un acercamiento a su empleo en el biocontrol de insectos lepidópteros que son plagas agrícolas. Revista Argentina de Microbiología 40(2):124-140.

Sauka, D.H., R.E. Basurto-Ríos, J.E. Ibarra, and G.B. Benintende. 2010. Characterization of an Argentine isolate of Bacillus thuringiensis similar to the HD-1 strain. Neotropical Entomology 39(5):767-773. doi:10.1590/S1519566X2010000500016.

Sauka, D.H., and G.B. Benintende. 2017. Diversity and distribution of lepidopteran-specific toxin genes in Bacillus thuringiensis strains from Argentina. Revista Argentina de Microbiología 49(3):273-281. doi:10.1016/j. ram.2017.02.003.

Schnepf, E., N. Crickmore, J. Van Rie, D. Lereclus, J. Baum, J. Feitelson, et al. 1998. Bacillus thuringiensis and its pesticidal crystal proteins. Microbiol. Mol. Biol. Rev. 62:775-806.

Souza, A.P., M.R. Marques, T.S. Mahmoud, B.A. Caputo, G.M. Canhete, C.B. Leite, et al. 2008. Bioprospecting insecticidal compounds from plants native to Mato Grosso do Sul, Brazil. Acta Bot. Bras. 22(4):1136-1140.

Stewart, G.S.A.B., K. Johnstone, E. Hagelberg, and D.J. Ellar. 1981. Commitment of bacterial spores to germinate. Biochem. J. 198:101-106.

Theunis, W., R.M. Aguda, W.T. Cruz, C. Decock, M. Peferoen, B. Lambert, et al. 1998. Bacillus thuringiensis isolates from the Philippines: habitat distribution, $\delta$-endotoxin diversity, and toxicity to rice stem borers (Lepidoptera: Pyralidae). Bulletin of Entomological Research 88:335-342.

Vázquez-Ramírez, M.F., J.C. Rangel-Núñez, J.E. Ibarra y M.C. Del Rincón-Castro. 2015. Evaluación como agentes de control biológico y caracterización de cepas mexicanas de Bacillus thuringiensis contra el gusano cogollero del maíz Spodoptera frugiperda (Lepidotera: Noctuidae). Interciencia 40(6):397-402.

Vilas-Bôas, G.T., A.P. Peruca, and O.M. Arantes. 2007. Biology and taxonomy of Bacillus cereus, Bacillus anthracis and Bacillus thuringiensis. Can. J. Microbiol. 53(6):673-687. 